\title{
Exposure to PM2.5, ultrafine particle and lung function among photocopy workers in Selangor
}

\begin{abstract}
This study investigates the relationship between exposure to PM2.5 and ultrafine particle (UFP) with respiratory health among photocopy workers in Selangor. This cross-sectional comparative study involved 60 workers where 30 photocopy workers as exposed group and 30 administrative staff as comparative group. Questionnaire adapted from American Thoracic Society (ATS) was used to assess respiratory symptoms. Lung function test was conducted for each respondent to assess lung function status. Measurements of PM2.5 and ultrafine particle were performed to obtain exposure level of the respondents. The mean personal exposure to PM2.5 (62.30 عg/m3) was five times higher and UFP (14567.10 pt/cc) was four times higher in exposed group. Reported respiratory symptoms of cough $(26.7 \%)$, phlegm $(16.7 \%)$, chest tightness $(3.3 \%)$ and wheezing $(6.7 \%)$ were higher in exposed group compared to the comparative group. There was a significant association between exposure to PM2.5 with lung function of FVC \% predicted $(r=i 0.404, p=0.027)$ and UFP with lung function of FEV1 \% predicted $(\mathrm{r}=0.377, \mathrm{p}=0.040)$. Continuous exposure to PM2.5 and UFP among photocopy workers can cause lung function impairment as this study showed that respiratory symptoms was higher among exposed group compared to the comparative group. There was a significant association between personal exposures to PM2.5 and UFP with lung function among exposed group. Emission and exposure to PM2.5 and UFP can be reduced by regular service or maintenance. Good ventilation also will ensure improved indoor air environment.
\end{abstract}

Keyword: Photocopiers; Ultrafine particle (UFP); PM2.5; Lung function 